\title{
Cultivable Microbiota in Mitis Salivarius Agar from Dental Plaque of Dogs
}

\author{
Fábio Alessandro Pieri ${ }^{1, ~ *, ~ V i t o r ~ d e ~ O l i v e i r a ~ S i l v a ~}{ }^{2}$, Abelardo Silva Junior ${ }^{2}$, \\ Maria Aparecida Scatamburlo Moreira ${ }^{2}$ \\ ${ }^{1}$ Basic Life Sciences Department, Life Sciences Institute, Federal University of Juiz de Fora, Governador Valadares, Brazil \\ ${ }^{2}$ Veterinary Department, Health and Biological Sciences Center, Federal University of Viçosa, Viçosa, Brazil \\ Email address: \\ fabio.pieri@ufjf.edu.br (F. A. Pieri) \\ ${ }^{*}$ Corresponding author
}

\section{To cite this article:}

Fábio Alessandro Pieri, Vitor de Oliveira Silva, Abelardo Silva Junior, Maria Aparecida Scatamburlo Moreira. Cultivable Microbiota in Mitis Salivarius Agar from Dental Plaque of Dogs. Animal and Veterinary Sciences. Vol. 6, No. 2, 2018, pp. 21-26.

doi: 10.11648/j.avs.20180602.11

Received: April 6, 2018; Accepted: April 23, 2018; Published: May 15, 2018

\begin{abstract}
Periodontal disease, which affects the support structures and protection of teeth, is the main disease found in dogs, reaching about $85 \%$ prevalence in animals over 4 years old. The most important bacteria in the initial colonization of human dental plaque are Streptococcus, which produce exopolysaccharides that facilitate fixation of the bacteria to the surfaces. Some studies have sought to identify the dental plaque microbiota in dogs using non-selective culture media, and have reported small amounts of Streptococcus, however they did not provide ideal conditions for the growth of this bacteria. The aim of this study was to isolate and identify the initial dental plaque microbiota of dogs cultivable in a selective medium for Streptococcus, and verify the presence of this genus. Genotypic identification of the isolates were carried out by partial $16 \mathrm{~S}$ rDNA sequencing. A total of 179 strains were obtained from the plaque of $10 \mathrm{dogs}$ without periodontal disease. The technique employed allowed 15 different microbial groups to be isolated, including the genus Streptococcus. This represented 33 (18.4\%) of 179 isolates, thus illustrating the partially selective ability of Mitis Salivarius Agar for samples of dental plaque in dogs.
\end{abstract}

Keywords: Dogs, Oral Microbiology, Dental Plaque, Microbiota, Bacteria, 16S rDNA

\section{Introduction}

Periodontal disease, which affects the support structures and protection of teeth, is a disease that is the mostly found in dogs, reaching about $85 \%$ prevalence in animals over the age of 4 years [1]. The etiologic agent of this disease is the bacterial plaque which develops on the tooth surface and the of immune system's reaction to these agents. Plaque builds up with the initial colonization of the tooth surface by indigenous bacteria, and promotes the adhesion of other, more virulent bacteria [2].

In humans, the microbial profile of plaque is been defined. It is formed predominantly by Gram-positive, aerobic, nonmotile and anaerobic bacteria in the early stages of infection $[3,4]$, but by Gram negative and mobile bacteria in the later stages of infection and inferred that the same microbial profile occurs in canine plaque [5].
The most important bacteria in the initial colonization of human dental plaque are Streptococcus [3], which produce exopolysaccharides that facilitate fixation of the bacteria to the surfaces, especially in places where minor irregularities occur, such as fissures or roughness [5]. Some studies have sought to identify the dental plaque microbiota in dogs using non-selective culture media, and have reported small amounts of Streptococcus [6-8]. However, since special conditions are required to isolate this kind of bacteria from samples with a diverse microbiota, its possible that it growth was inhibited by other bacteria. Other work presented low presence of Streptococcus spp. in enamel surface of dogs, performing the identification without culture techniques, using pyrosequencing of $16 \mathrm{~S}$ rDNA, and presented bacteria of Neisseria, Moraxella and Bergeyella species as the main 
bacterial groups in this environment [9]. However in human studies is presented the need to use a selective medium such as Mitis Salivarius Agar to obtain higher presence of Streptococcus spp., from dental plaque samples [10].

Thus, the importance of the genus Streptococcus in the composition of canine dental plaque remains unknown. Such knowledge is essential to determining therapeutic procedures for the treatment or prevention of periodontal disease in dogs. The aim of this study was, therefore, to isolate and identify the initial dental plaque microbiota of dogs cultivable in a selective medium for Streptococcus and to verify the presence of this genus in the samples.

\section{Materials and Methods}

\subsection{Animals and Sampling}

Bacterial isolates were obtained from the supragingival plaque of 10 mongrel dogs aged between 1 and 2 years, without clinical signs of periodontal disease, periodontal pockets, gingival swelling or redness. All the animals were from the experimental kennels of the Department of Veterinary Medicine or treated at the Veterinary Hospital of the Federal University of Viçosa. All procedures complied with the rules established by the Ethics Committee in the use of animals at the Federal University of Viçosa, and were approved by the same protocol under No. 18/2010.

\subsection{Sample Processing}

The collection of dental plaque was performed using a modified version of the method described by [8]. Plaque was obtained from the fourth premolars of $10 \mathrm{dogs}$ by friction, with the aid of sterile swabs. Following collection, the swabs were transferred to tubes containing a solution of Tris- $\mathrm{HCl}, \mathrm{pH}$ 7.2. Glass beads were introduced and the tubes were placed in a vortex model MS1 minishaker (IKA Laboratory Equipment, Artur Nogueira, Brazil) at 2200 rpm for 2 minutes.

Suspensions were serially diluted on a decimal scale to 1:10000. All were inoculated on the surface of plates containing $20 \mathrm{ml}$ Mitis Salivarius Agar (Himedia, Mumbai, India) and potassium tellurite $1 \%$, prior to incubation at $37^{\circ} \mathrm{C}$ for 72 hours in microaerophilic environmet, in order to provide optimal conditions for the isolation of Streptococcus sp. using BBL GasPak Plus Anaerobic System Envelopes with Palladium Catalyst kit (BD Biosciences, New Jersey, USA).

After incubation, colonies with different morphological characteristics were selected and streaked on Brain Heart Infusion agar (BHI) (Himedia, Mumbai, India), after which they were again incubated, this time at $37^{\circ} \mathrm{C}$ for 24 hours, in order to obtain pure cultures. Following purification, one colony from each culture was transferred to a tube containing $\mathrm{BHI}$ and once again incubated for 24 hours at $37^{\circ} \mathrm{C}$, at which point glycerol $(20 \% \mathrm{v} / \mathrm{v})$ was added and the cultures were frozen in liquid nitrogen and maintained at $-80^{\circ} \mathrm{C}$.

To subsequently activate the cultures, a $10 \mu \mathrm{L}$ aliquot of each stock was transferred to a BHI broth and incubated at $37^{\circ} \mathrm{C}$ for 24 hours.

\subsection{Phenotypic Determinations}

Upon reactivation in $\mathrm{BHI}$ broth, the isolates were subjected to Gram staining and tested for catalase production as described by American Society for Microbiology [11, 12].

\subsection{Polymerase Chain Reaction - PCR}

DNA was extracted using a Wizard ${ }^{\circledR}$ Genomic DNA Purification Kit (Promega Corporation, Madison, USA) according to the manufacturer's protocol. DNA extraction was performed using $1.5 \mathrm{~mL}$ of the activated culture. The DNA was purified using a Genomic DNA Kit ${ }^{\mathrm{TM}}$ GeneJET Purification (Fermentas International Inc., Maryland, USA) according to the manufacturer's protocol, eluted with $70 \mu \mathrm{L}$ of water and stored at $-20^{\circ} \mathrm{C}$. DNA from Streptococcus salivarius CDC 262 was used as a positive control in the PCR and Nuclease-Free Water (Promega Corporation, Madison, USA) was used as a negative control.

Genotypic identification of the isolates was carried out by partial 16S rDNA sequencing, using the primer pair $616 \mathrm{~V}\left(5^{\prime}-\right.$ AGAGTTTGATYMTGGCTCA-3') and 630R (5'AAGGAGGTGATCCARCC-3'), binding at positions 11-26 and 1525-1541 of the 16S rDNA, respectively [13], in order to produce an amplicon of approximately 1530 base pairs. The primers were commercially synthesized by Integrated DNA Technologies Inc. (Iowa, USA). Polymerase chain reaction (PCR) was performed using PCR Master Mix (Promega Corporation, Madison, USA) according to the protocol used by Sterr et al. [13]. The PCR was conducted in a thermal cycler (Axygen Scientific, California, USA). The amplified fragments were visualized by electrophoresis on $1 \%$ agarose gel (Invitrogen) using GelRed ${ }^{\mathrm{TM}}$ Nucleic Acid Stain (6X) (Biotium Inc., California, USA) and the UV image capturing system in LPIX HE (Loccus Biotecnologia, São Paulo, Brazil).

\subsection{Sequencing and Data Analysis}

Amplified fragments of expected size, together with the positive control, were sent for sequencing by the Macrogen Company (in Korea), which used the method previously described by Sanger et al. [14].

The sequences obtained were edited using Sequencher software (version 4.1.4). All isolates were initially identified by performing database searches, comparing $16 \mathrm{~S}$ rDNA sequences obtained from a single reading with other related sequences in GenBank, using the Basic Local Alignment Software Search Tool (BLAST), available from the National Center for Biotechnology Information - NCBI (http://www.ncbi.nlm.nih.gov). Multiple alignments were obtained using Clustal W2 software (http://www.ebi.ac.uk/Tools/clustalw2/index.html), from which a phylogenetic tree was also constructed using Molecular Evolutionary Genetics Analysis (MEGA) software (version 4.0 for Windows; www.megasoftware.net) by means of the Unweighted Pair Group Method with Arithmetic Mean (UPGMA). Statistical analysis was performed using 2000 bootstrap replicates. We used a sequence of Sulfolobus sp. [GenBank: FJ489515.1] 16S rDNA as the outgroup. A 
difference of $3 \%$ or greater was taken to indicate that the sequences were probably from distinct species [15].

\subsection{Nucleotide Sequence Accession Numbers}

The nucleotide sequences determined in this study have been deposited in GenBank [GenBank: HQ717175 to HQ717353].

\section{Results}

A total of 179 strains were obtained from the plaque of 10 dogs without periodontal disease (Figure 1). As expected, Gram staining indicated $90 \%$ positive cocci and rods, representing 164 isolates. These were $66 \%$ catalase negative. The 179 isolates had fragments of $16 \mathrm{~S}$ rDNA amplified and sequenced. The sequences obtained were deposited in GenBank (Supplementary Material 1 and 2) and identified by comparison with other gene sequences from the same database. Of the 179 isolates sequenced, 46 were identified to species level, while the other 133 were not (data not shown).

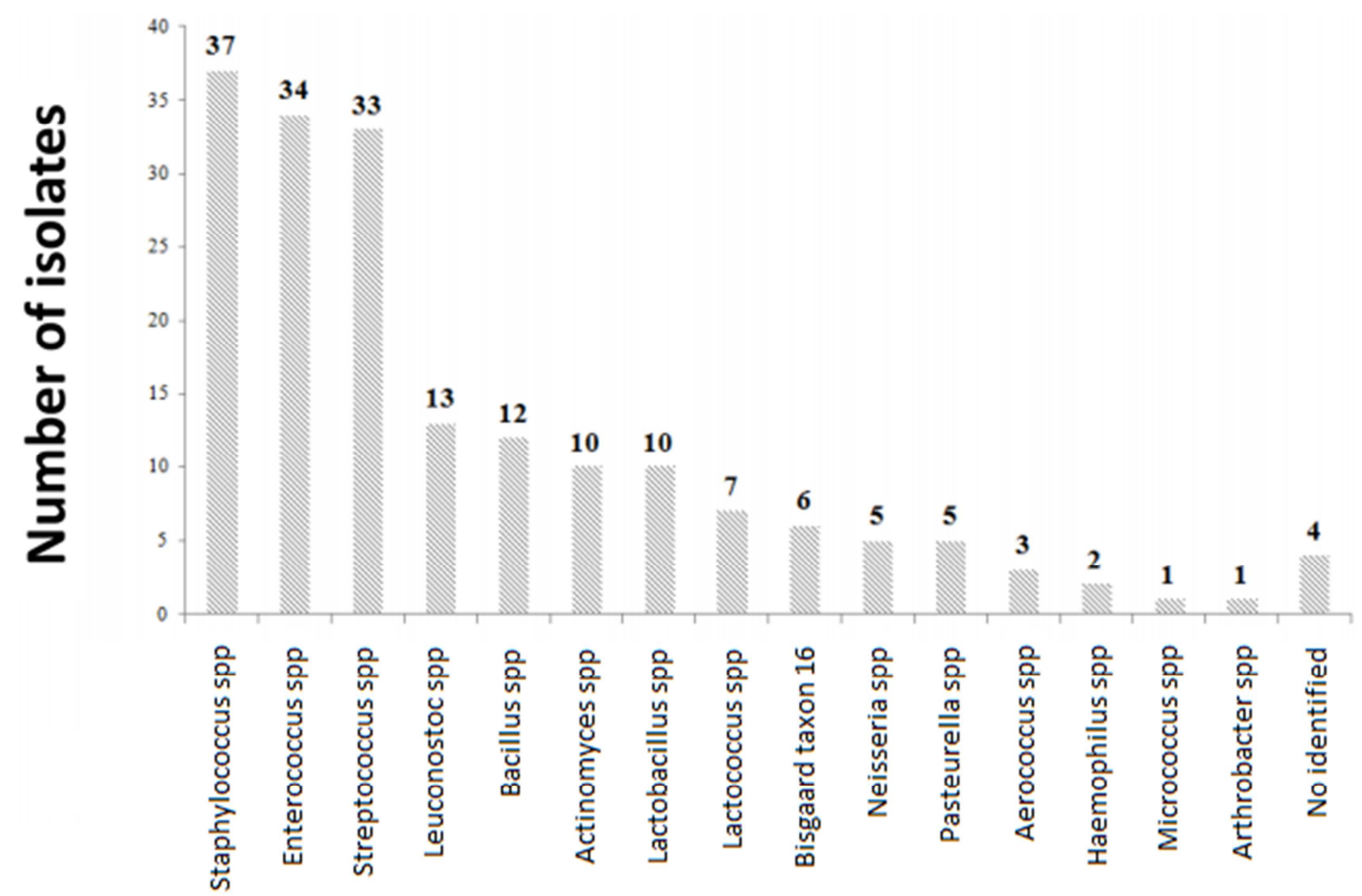

Figure 1. Identification to genera level of the 179 isolates obtained from the dental plaque of dogs based on sequencing of the $165 \mathrm{rDNA}$ fragment.

Despite the targeting of the isolation technique for the genus Streptococcus, the bacterial diversity found in this study indicated a total of 14 genera belonging to three phyla. The genera Aerococcus, Bacillus, Lactobacillus, Lactococcus, Leuconostoc, Enterococcus, Staphylococcus and Streptococcus represented the phylum Firmicutes (low G+C, Gram-positive), Actinomyces, Arthrobacter and Micrococcus represented the phylum Actinobacteria (high $\mathrm{G}+\mathrm{C}$, Gram-positive), and Neisseria, Haemophilus and Pasteurella represented the phylum Proteobacteria (Gramnegative). A total of six isolates of the bacteria known as Bisgaard taxon 16 [16] were also found in two dogs.

The bacterial profile constructed from the dental plaque isolates obtained from the dogs is shown as a phylogenetic tree (Figure 2). Streptococcus sp., 33/179 (18.4\%), Staphylococcus sp. 37/179 (20.7\%) and Enterococcus sp., $34 / 179(19 \%)$ were the prevalent genera in the dental plaque stages (Figure 1). These genera were found in $9 / 10(90 \%)$, $8 / 10(80 \%)$ and $5 / 10(50 \%)$ of the dogs respectively, representing $105 / 179(58.6 \%)$ of the total number of isolates.

\section{Discussion}

The technique employed in this study not only enabled the isolation of bacteria of the genus Streptococcus, but also other bacteria (Figure 1), including rods and Gram-negative, demonstrating the partially selective capability of the Mitis Salivarius Agar. Among the main genera isolated, Streptococcus was the third most common, surpassed by both Staphylococcus and Enterococcus (Figure 1).

Elliot et al. [6] identified the microbiota in the subgingival plaque of 10 dogs, with Streptococcus sp. representing only $1 \%$ of the total. Those investigations, however, utilized plates of a different nature and different culture media from those employed in the present study. From a total of 32 isolates obtained from dogs without gum disease using non-selective culture media, Riggio et al. [8] identified only one isolate of Streptococcus minor, which was also identified in this study, as well as in other studies examining plaque from dogs and cats $[6,17,18]$. 


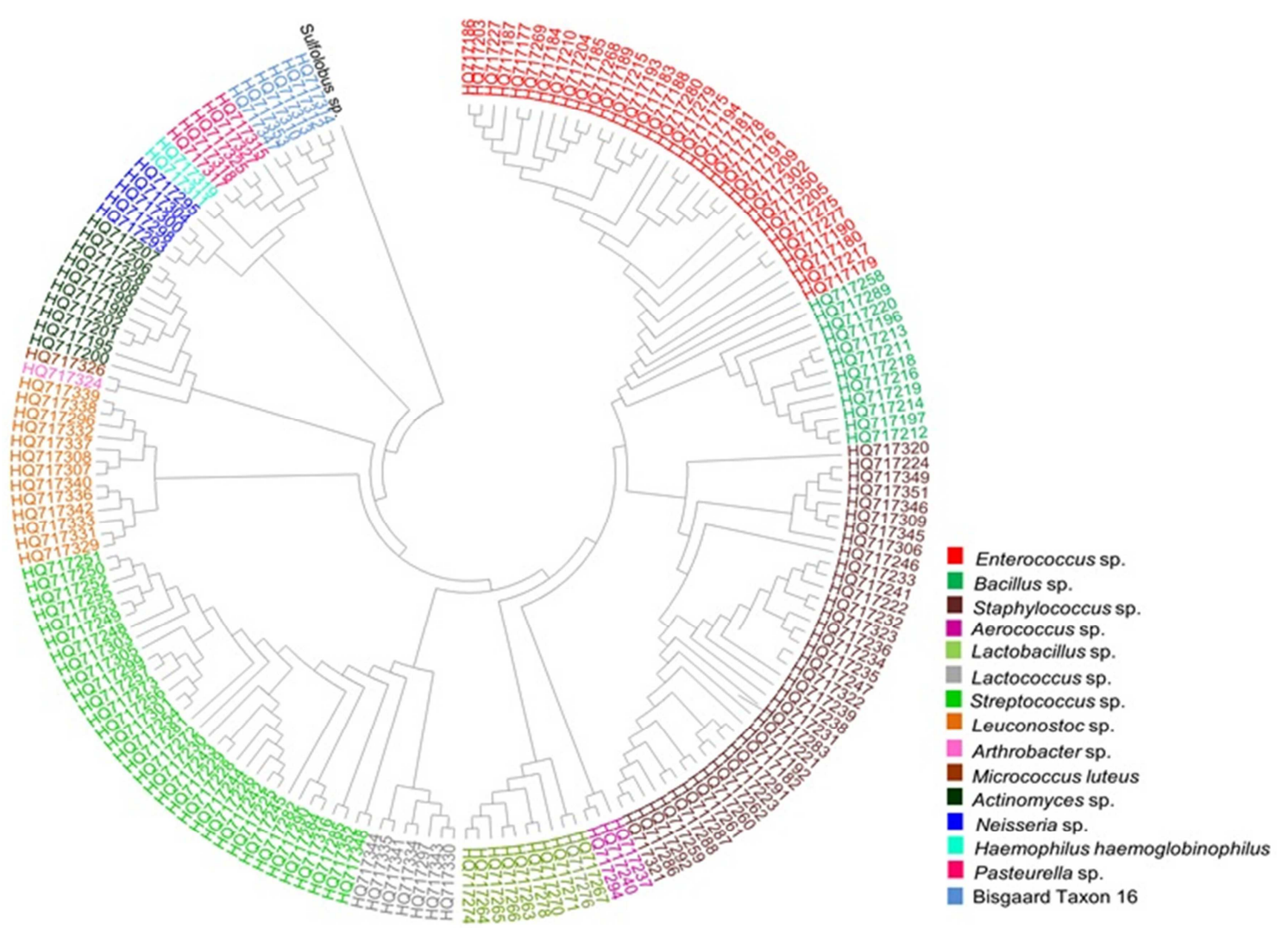

Figure 2. Phylogenetic Unweighted Pair Group Method with Arithmetic Mean (UPGMA) dendrogram showing all isolates for which sequences were obtained from ten dogs aged between one and two years identified by $16 \mathrm{~S}$ rDNA sequencing. Sequences are identified by their access numbers in GenBank. 179 sequences are included in the tree.

Takada et al. [10] were able to isolate Streptococcus using Mitis Salivarius Agar with supplements, with a recovery rate of $21.2 \%$ of all isolates. They also reported that the medium removed $89.7 \%$ of total bacteria grown in $\mathrm{BHI}$ from the same blood sample. The main species identified by Takada et al. [10] were S. mitis and S. sanguis, demonstrating the bias of this medium towards the growth of Streptococcus.

It is noteworthy that none of the above studies was able to isolate $S$. mutans, which is considered the most important bacteria in human plaque formation. This corroborates the observation of Dent and Marsh [19] that there is a correlation between the presence of $S$. mutans and diets rich in sucrose, which is not the case with dogs. Takada et al. [10] suggest that the different $\mathrm{pH}$ of a dog's oral cavity, compared to that of a human, may be a factor responsible for the absence of this type bacteria in these animals.

Studies on the microbiota of supragingival plaque from beagle dogs, assessed using DNA-DNA hybridization, identified several Streptococcus species, including some associated with dental plaque microbiota in humans, such as S. mitis, S. constelattus, S. oralis and S. sanguis [20]. These species were not isolated in the current study, since the sequences of Streptococcus sp. obtained showed no similarity with the examples of these species deposited in GenBank. Controversially, Holcombe et al. [9] did not presented significant presence of Streptococcus spp., in enamel dental plaques of 12 dogs with initial dental plaque formation between 24 and 48 hours after dental professional cleaning, which could suggest that the presence of this bacterial group could be increased just after later events in the development of dental plaque in animals.

The difficulty in identifying bacteria to the species level, especially when dealing with the genera Staphylococcus, Streptococcus and Enterococcus, can be because closely related species may have virtually identical $16 \mathrm{~S}$ rDNA gene sequences. Some species of Streptococcus group D, for example, share a $99 \%$ similarity in their $16 \mathrm{~S}$ rDNA sequence [21].

Bacteria of the genera Actinomyces, Porphyromonas, Fusobacterium, Neisseria, Staphylococcus, Streptococcus and Veillonella were isolated in most samples analyzed by Dent and Marsh [19], Kim et al. [22], Zambori et al. [23] and Jeussete et al. [24], suggesting that representatives of these genera can be considered as characteristic or indigenous members of the microbial community in dental plaque. This was confirmed in the present study, which verified the presence of the genera Staphylococcus, Streptococcus, Actinomyces and Neisseria (as shown in Figure 1). As expected, the genera Porphyromonas, Fusobacterium and Veillonella, found in other works [19, 22, 23, 24] were not isolated as result of the methodology applied and the nutritional and strict anaerobic needs of these bacteria.

Study by Nowroozilarki et al. [25], presented Helicobacter spp. and Wolinella spp., in oral cavities of toy breed dogs, 
different of our findings. However, the dog population studied differ from that used in our work. In that work the aim was to study dogs older from six years with periodontal disease, meanwhile in the present study were used young dogs without oral diseases, suggesting that the inclusion of that genera [25] occur in a new environment provided in oral dental plaque of older dogs by the evolution of that specific oral disease.

It is suggested that Bisgaard taxon 16 [16], that has not yet formally referred in the bacterial taxonomy, it is part of oral microflora of dogs, since the few reports of them were obtained from this site $[6,16]$ and in this study, it was obtained from two different dogs.

The sequence analyses and literature review indicated that $59 / 179(33 \%)$ of the bacteria isolated from dogs in this study are not normally found in human oral cavities [26, 27]. Only $5 / 15(33.3 \%)$ of the identified groups (14 genera plus Bisgaard taxon 16), namely Actinomyces, Neisseria, Haemophilus, Streptococcus and Lactobacillus, are considered members of human dental supragingival plaque $[22,23]$.

Recent works [23, 28-30] presented the sensitivity profile of some isolates presented in this work when challenged by natural compounds from vegetable and other bacteria, with important findings in the study of dental plaque control in dogs. These studies have to be conducted to confirm these alternative therapic drugs to replace chlorhexidine use, that presents a set of side effects on its long-term use [5].

\section{Conclusions}

It follows that the technique employed in this study allowed 15 different microbial groups to be isolated, including the genus Streptococcus, which represented $18.4 \%$ of 179 isolates. This demonstrates the partially selective ability of Mitis Salivarius Agar when analyzing samples of dental plaque from dogs. In addition the results indicated that the genus Streptococcus spp. bacteria can be present in higher levels in dogs dental plaque environment, particularly those included in group D streptococci.

\section{Acknowledgements}

The authors thank the Foundation for Research Support of the State of Minas Gerais (FAPEMIG) for financial support by the project funding registered by the number APQ-0149509 and the Brazilian Coordination of Improvement of Higher Education Personnel (CAPES) for the doctoral scholarship of Fabio Alessandro Pieri between 2009 and 2012.

\section{References}

[1] Kyllar, M. and Witter, K. (2005). Prevalence of dental disorders in pet dogs. Veterinary Medicine 11:496-505.

[2] Niemiec, B. A. (2008). Periodontal therapy. Topics in Companion Animals Medicine 23:81-90.
[3] Rosan, B. and Lamont, R. J. (2000). Dental plaque formation. Microbes Infection 2:1599-1607.

[4] Komori, R., Sato, T., Takano-Yamamoto, T. and Takahashi, N. (2012). Microbial composition of dental plaque microflora on first molars with orthodontic bands and brackets, and the acidogenic potential of these bacteria. Journal of Oral Bioscience 54:107-112.

[5] Pieri, F. A., Daibert, A. P., Bourguignon, E., Moreira, M. A. S (2012). Periodontal disease in dogs. In: Perez-Marin, C. C. A Bird's-Eye View of Veterinary Medicine. InTech, Zagreb, Croatia, pp: 119:140.

[6] Elliott, D. R., Wilson, M., Buckley, C. M. and Spratt, D. A. (2005). Cultivable oral microbiota of domestic dogs. Journal of Clinical Microbiology 43:5470-5476.

[7] Fonseca, S. A. D., Galera, P. D., Brito, D. L., Perecmanis, S., Silva, A. S., Cardoso, L. B., Marçola, T. G., Drummond, V. O and Pimentel, C. M. (2011). Análise microbiológica da placa bacteriana da doença periodontal em cães e o efeito da antibioticoterapia sobre ela. Ciência Rural 41:1424-1429.

[8] Riggio, M. P., Lennon, A., Taylor, D. J. and Bennett, D. (2011). Molecular identification of bacteria associated with canine periodontal disease. Veterinary Microbiology, 150:394-400.

[9] Holcombe, L. J., Patel, N., Colyer, A., Deusch, O., O’Flynn, C. and Harris, s. (2014). Early Canine Plaque Biofilms: Characterization of Key Bacterial Interactions Involved in Initial Colonization of Enamel. PLOS one, 9:e113744.

[10] Takada, K., Hayashi, K., Sasaki, K., Sato T. and Hirasawa, M. (2006). Selectivity of Mitis Salivarius agar and a new selective medium for oral streptococci in dogs. Journal of Microbiological Methods, 66:460-465.

[11] Smith, A. C. and Hussey, M. A. (2013). Gram stain protocols. In: ACM Microbelibrary - Laboratory protocols. American Society for Microbiology, Washington, USA, Available in: http://www.microbelibrary.org/component/resource/gramstain/2886-gram-stain-protocols.

[12] Reiner, K. (2013). Catalase test protocol. In: ACM Microbelibrary - Laboratory protocols. American Society for Microbiology, Washington, USA, Available in: http://www.microbelibrary.org/component/resource/gramstain/2886-gram-stain-protocols.

[13] Sterr, Y., Weiss, A., and Schmidt, H; (2009). Evaluation of lactic acid bacteria for sourdough fermentation of amaranth. International Journal of Food Microbiology 136:75-82.

[14] Sanger, F., Nicklen, S. and Coulson, A. R. (1977). DNA sequencing with chain-terminating inhibitors. Proceedings of National Academy of Science of United States of America 74:5463-5467.

[15] Forney, L. J., Zhou X. and Brown, C. J. (2004). Molecular microbial ecology: land of the one-eyed king. Current Opinion in Microbiology 7:210-220.

[16] Bisgaard, M. and Mutters, R. (1986). Characterization of Some Previously Unclassified Pasteurella spp Obtained from the Oral Cavity of Dogs and Cats and Description of a New Species Tentatively Classified with the Family Pasteurellaceae Pohl-1981 and Provisionally Called Taxon-16. Acta Pathologica, Microbiologica et Imunologica Scandinavica B 94:177-184. 
[17] Vancanneyt, M., Devriese, L. A., Graef, E. M., Baele, M., Lefebvre, K., Snauwaert, C., Vandamme, P., Swings J. and Haesebrouck, F. (2004). Streptococcus minor sp. nov., from faecal samples and tonsils of domestic animals. International Journal of Systematic Evolutional Microbiology 54:449-452.

[18] Iijima, D. (2009). The Distribution of Genus Streptococcus in the Oral Cavities of Cats. International Journal of OralMedical Science 8:19-23.

[19] Dent, V. E. and Marsh, P. D. (1981). Evidence for a basic plaque microbial community on the tooth surface in animals. Archieves of Oral Biology 26:171-179.

[20] Rober, M., Quirynen, M., Haffajee, A. D., Schepers E. and Teughels, W. (2008). Intra-oral microbial profiles of beagle dogs assessed by checkerboard DNA-DNA hybridization using human probes. Veterinary Microbiology 127:79-88.

[21] Fox, G. E., Wisotzkey J. D. and Jurtshuk P. Jr. (1992). How close is close: 16S rRNA sequence identity may not be sufficient to guarantee species identity. International Journal Systematic Evolutionary Microbiology 42:166-170.

[22] Kim, S. E., Hwang, S. Y., Jeong, M., Lee, Y., Lee, E. R., Park, Y. W., Ahn, J. S., Kim, S. and Seo, K. (2016). Clinical and microbiological effects of a subantimicrobial dose of oral doxycycline on periodontitis in dogs. The Veterinary Journal 208: 55-59.

[23] Zambori, C., Morvay, A. A., Sala, C., Licker, M., Gurban, C., Tanasie, G. and Tirziu, E. (2016). Antimicrobial effect of probiotics on bacterial species from dental plaque. The Journal of Infection in Developing Countries 10:214-221.

[24] Jeussete, I. C., Román, A. M., Torre, C., Crusafont, J., Sánchez, N., Sánchez, M. C., Péres-Salcedo, L. and Herrera,
D. (2016). 24-hour evaluation of dental plaque bacteria and halitosis after consumption of a single placebo or dental treat by dogs. American Journal of Veterinary Research 77:613619.

[25] Nowroozilarki, N., Jamshidi, S., Zalehi, T. Z. and Kolahian, S. (2017) Identification of Helicobacter and Wolinella spp. in Oral Cavity of Toy Breed Dogs With Periodontal Disease. Topics in Compannion Animals Medicine 32:96-99.

[26] Paster, B. J., Boches, S. K., Galvin, J. L., Ericson, R. E., Lau, C. N., Levanos, V. A., Sahasrabudhe A. and Dewhirst, F. E. (2001). Bacterial diversity in human subgingival plaque. Journal of Bacteriology 183:3770-3783.

[27] Socransky, S. S. and Haffajee, A. D. (2005). Periodontal microbial ecology. Periodontology 2000 38:135-187.

[28] Lindinger, M. I. (2016). Reduced Dental Plaque Formation in Dogs Drinking a Solution Containing Natural Antimicrobial Herbal Enzymes and Organic Matcha Green Tea. Scientifica 2016: ID2183623.

[29] Pieri, F. A., Souza, M. C. C., Vermelho, L. L. R., Vermelho, M. L. R., Perciano, P. G., Vargas, F. S., Borges, A. P. B., Veiga-Junior, V. F., and Moreira, M. A. S. (2016). Use of $\beta$ caryophyllene to combat bacterial dental plaque formation in dogs. BMC Veterinary Research 12:216.

[30] Pieri, F. A., Silva, V. O., Vargas, F. S., Veiga-Junior, V. F. and Moreira, M. A. S. (2014) Antimicrobial activity of Copaifera langsdorffii oil and evaluation of its most bioactive fraction against bacteria of dogs dental plaque. Pakistan Veterinary Journal 34:165-169. 